\title{
Environmentally Controlled Charge Carrier Injection Mechanisms of Metal/WS 2 Junctions
}

\author{
Rik van Bremen, ${ }^{* \dagger} \dagger$ Kevin Vonk, ${ }^{\dagger}$ Harold J. W. Zandvliet, ${ }^{\dagger}$ and Pantelis Bampoulis ${ }^{*,+, \dagger}$ \\ ${ }^{\dagger}$ Physics of Interfaces and Nanomaterials, MESA+ Institute for Nanotechnology, University of Twente, P.O. Box 217, 7500 AE \\ Enschede, The Netherlands \\ ${ }^{\ddagger}$ Physikalisches Institut, Universität zu Köln, Zülpicher Straße 77, 50937 Köln, Germany
}

Supporting Information

\begin{abstract}
Here we report on a novel, noninvasive route for operando tailoring of the charge transport properties of metal/ $\mathrm{WS}_{2}$ contacts without the negative impacts to twodimensional materials arising from conventional doping methods. The doping level of thin $\mathrm{WS}_{2}$ flakes supported on insulating mica is susceptible to local charge variations induced by the presence of a hydration layer between mica and $\mathrm{WS}_{2}$. We demonstrate, via the use of several complementary scanning probe techniques, that the direct control of the state and thickness of this intercalated water film controls the charge injection properties of $\mathrm{Pt} / \mathrm{WS}_{2}$ nanocontacts. A switch from unipolar to ambipolar transport was achieved by environmentally controlling the thickness of the intercalated water. We show that the effect persists even for multilayer flakes and that it is completely reversible, opening a new route toward the realization of novel electronics with environmentally controllable functionalities.
\end{abstract}

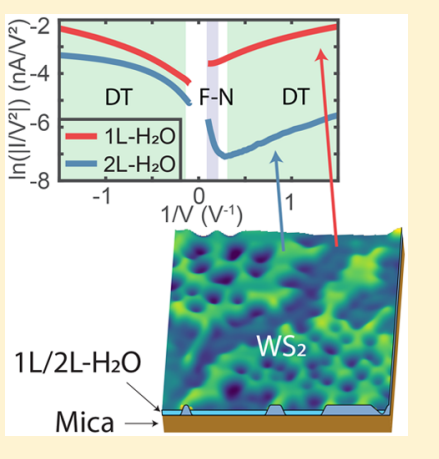

A

major challenge when incorporating two-dimensional (2D) materials in devices is the control of the contact properties of the metallic leads ${ }^{1-6}$ and the influence of atmospheric conditions on these contact properties. $^{7-11}$ For $2 \mathrm{D}$ semiconductors, the transport behavior of devices is often determined by the metal-semiconductor junction. In the Schottky-Mott model, the barrier height for charge carrier injection in such a junction is determined by the difference between the Fermi level of the metal and the electron affinity of the semiconductor. However, Fermi level pinning and metalization of the semiconductor often make it difficult to control the barrier height via the metal contact. ${ }^{12-14}$ It has been shown that Fermi level pinning can be alleviated by using transferred atomically flat metal contacts ${ }^{15}$ or by the use of $2 \mathrm{D}$ buffer layers. ${ }^{16-18}$ Vacuum deposition of $\mathrm{Au}$ contacts $^{19}$ and annealing in vacuum ${ }^{20}$ have also resulted in lower contact resistances. Another promising method to control the contact properties is the adjustment of the Fermi level of the 2D semiconductor. ${ }^{21}$ Unfortunately, shifting the Fermi level by incorporating dopants in an atomically thin layer is not as successful as it is in bulk semiconductors as the embedding of dopants with traditional techniques induces damage to the $2 \mathrm{D}$ semiconductor. $^{22}$

$\mathrm{WS}_{2}$ is one of the members of the transition-metal dichalcogenide (TMDC) family with semiconducting properties. As a bulk crystal it has an indirect band gap of $1.3 \mathrm{eV}$, while single-layer $\mathrm{WS}_{2}$ has a direct band gap of $2.1 \mathrm{eV}^{23} \mathrm{~A}$ transistor of a single layer of $\mathrm{WS}_{2}$ shows mobilities up to 50 $\mathrm{cm}^{2} /(\mathrm{V} \mathrm{s})$ and a current on/off ratio of $10^{6}$. ${ }^{24}$ The electric properties of $\mathrm{WS}_{2}$ transistors manufactured on standard $\mathrm{Si} /$ $\mathrm{SiO}_{2}$ are strongly affected by charge puddles and ripples induced by the substrate. ${ }^{25,26}$ An alternative substrate is muscovite mica. Muscovite mica is atomically flat and therefore does not lead to rippling of the adlayer. ${ }^{27}$ This makes mica an ideal candidate for maintaining high carrier mobilities and high switching speeds of the adlayer. ${ }^{28}$ As we will show, another advantage of mica is its hydrophilicity, which promotes water adsorption at ambient conditions. ${ }^{29}$ These water films are still present between the mica and a supported $2 \mathrm{D}$ material. ${ }^{30}$ The thickness of the water film depends on the relative humidity (RH) and temperature of the environment. ${ }^{31,32}$ This way the water thickness can be varied from 1 to 3 layers. ${ }^{33-35}$

In this Letter we will show that the properties of a $\mathrm{Pt} / \mathrm{WS}_{2}$ contact can be controlled not only by the thickness of the $\mathrm{WS}_{2}$ flake $^{36}$ but also by the hydration state of the underlying supporting substrate. Static water doping of the $\mathrm{WS}_{2}$ layer is induced by charge transfer from the underlying substrate and further controlled by the exact thickness of the water film. ${ }^{37}$ We demonstrate that the charge carrier injection mechanism in $\mathrm{Pt} / \mathrm{WS}_{2}$ nanocontacts is directly controlled by the thickness of the intercalated water film. In ambient conditions, thermionic emission (TE) is identified to be the dominant injection mechanism in thicker $\mathrm{WS}_{2}$ flakes. At lower $\mathrm{RH}$, water doping leads to a substantial enhancement of Fowler-Nordheim (F$\mathrm{N})$ tunneling, increasing the reverse bias current. The contact properties of thinner $\mathrm{WS}_{2}$ flakes are well-described by a combination of $\mathrm{F}-\mathrm{N}$ and direct tunneling (DT). In particular, we show a shift from unipolar to ambipolar transport when reducing the thickness of the intercalated water (between thin $\mathrm{WS}_{2}$ and mica) from two layers to one layer. We show that

Received: March 26, 2019

Accepted: May 1, 2019

Published: May 1, 2019 

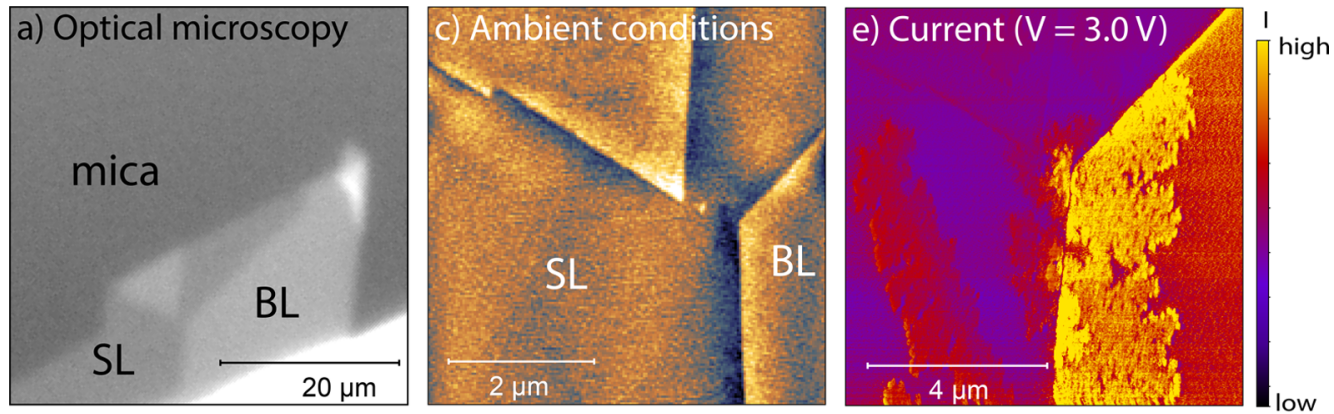

b) Experimental set-up
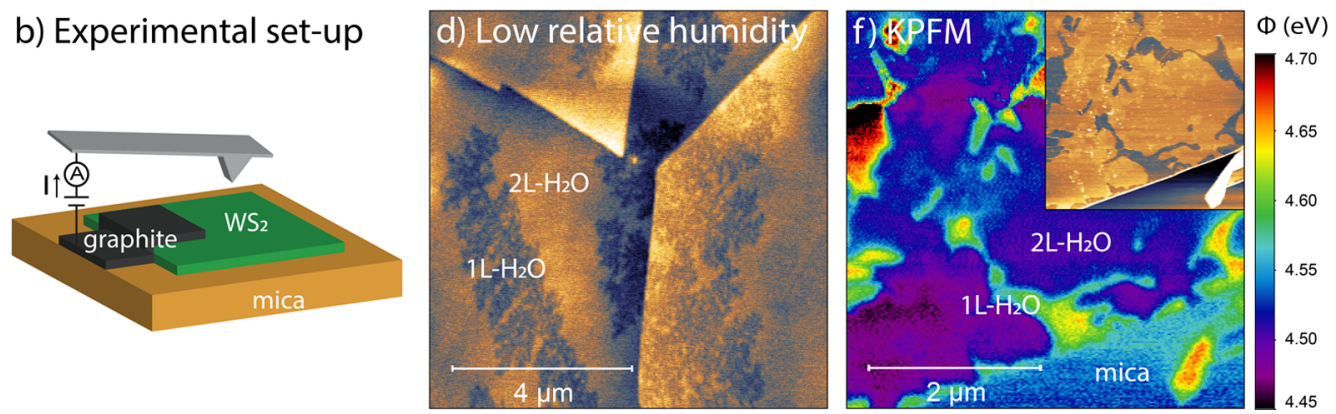

Figure 1. (a) Optical microscopy image of a thin $\mathrm{WS}_{2}$ flake placed on mica. The indicated SL and BL areas were identified by AFM. (b) Schematic representation of the experimental setup. (c) Contact mode AFM topography image of the SL and BL areas at ambient conditions. (d) The same area after reducing the relative humidity of the chamber. The intercalated water film evaporated partially, resulting in $1 \mathrm{~L}-\mathrm{H}_{2} \mathrm{O}$ ice fractals. (e) Current map, recorded simultaneously with the topography map of panel $\mathrm{d}$. The measured current is higher at the fractal regions as compared to its surrounding $2 \mathrm{~L}-\mathrm{H}_{2} \mathrm{O}$ regions. (f) Contact potential difference map. The simultaneously recorded topography is shown in the inset.

water doping can reduce the contact resistance and eliminate the unipolar properties of the contact. This method of controlling the local charge transfer from the supporting substrate is a nonintrusive doping approach, which retains the intrinsic physical properties of the 2D semiconductor. Furthermore, we demonstrate that this approach provides a novel pathway for the realization of environmentally controlled 2D TMDC-based devices with in situ tunability.

Figure 1a shows the optical micrograph of a thin $\mathrm{WS}_{2}$ flake on mica. To study the system with conductive-atomic force microscopy (c-AFM), we have made an electrical connection to the AFM tip by placing a graphite flake on the $\mathrm{WS}_{2}$, as shown in Figure 1b. A metallic lead is then connected to the graphite flake. AFM topography reveals that the flake consists of regions of single-layer (SL), bilayer (BL), and few-layer (FL) $\mathrm{WS}_{2}$, where the FL part is 10 layers thick. The determination of the $\mathrm{WS}_{2}$ flake thickness is described in detail in the Supporting Information, Figure S1. An AFM topography image of the regions containing $\mathrm{SL}$ and $\mathrm{BL} \mathrm{WS}_{2}$ is shown in Figure 1c. During the sample preparation, a water film is trapped between the mica and the $2 \mathrm{D}$ material. ${ }^{30}$ The water film is difficult to detect by AFM at ambient conditions because of its uniform thickness. Upon reduction of the relative humidity $(\mathrm{RH})$ to $<1 \%$ (hereby referred to as low $\mathrm{RH}$ ), fractal-shaped depressions become visible because of partial evaporation of the water layer through defects such as wrinkles and step-edges as seen in Figure 1d. ${ }^{32,38}$ Similar fractals trapped between mica and graphene were previously found to be due to a monolayer of ordered ice (termed 1L$\mathrm{H}_{2} \mathrm{O}$ ) surrounded by thicker regions of less ordered bilayer of water (termed $2 \mathrm{~L}-\mathrm{H}_{2} \mathrm{O}$ ). ${ }^{38}$ We emphasize that the notations $1 \mathrm{~L}-\mathrm{H}_{2} \mathrm{O}$ and $2 \mathrm{~L}-\mathrm{H}_{2} \mathrm{O}$ refer to one layer and two layers of water trapped between $\mathrm{WS}_{2}$ and mica. While the exact nature of these fractal structures is still under debate, ${ }^{31,32,38}$ the influence of these two different regions to the $2 \mathrm{D}$ cover is remarkable, as we will show shortly.

Earlier studies using graphene as the cover layer demonstrated that the fractal-shaped depressions lead to p-type doping of the graphene. ${ }^{37}$ This doping was previously attributed to charge transfer from the underlying negatively charged substrate, due to either the mica ${ }^{39}$ or the presence of an ordered intercalated water film. ${ }^{38}$ To determine the influence of the underlying water layer on the metal/ $\mathrm{WS}_{2}$ contact, we measured the conductivity of the tip/sample contact with the AFM probe. This is done by applying a bias voltage between the AFM tip and sample and measuring the resulting current during scanning. The measured conductivity is dominated by the tip/sample contact as the resistances of the channel and the graphite/ $\mathrm{WS}_{2}$ contact are both negligible. ${ }^{40}$ This way the c-AFM map of Figure 1e has been obtained simultaneously with the topography map of Figure $1 \mathrm{~d}$. The c-AFM map reveals indeed a difference between $\mathrm{WS}_{2}$ placed on $1 \mathrm{~L}-\mathrm{H}_{2} \mathrm{O}$ and $\mathrm{WS}_{2}$ placed on $2 \mathrm{~L}-\mathrm{H}_{2} \mathrm{O}$, with the $1 \mathrm{~L}$ $\mathrm{H}_{2} \mathrm{O}$ regions being more conductive than the $2 \mathrm{~L}-\mathrm{H}_{2} \mathrm{O}$ regions. The reason for this change in conductivity is due to the state of the intercalated water layer and will be explored in the remainder of this Letter. In the c-AFM image it can also be seen that the thickness of the $\mathrm{WS}_{2}$ flake has a profound influence on the measured conductivity, where the conductivity is higher for the BL flake compared to the SL flake. This trend is continued for thicker flakes, as shown in the Supporting Information. Still, it should be noted that for different flake thicknesses the qualitative influence of the intercalated water layer is the same: the conductivity on $1 \mathrm{~L}$ $\mathrm{H}_{2} \mathrm{O}$ regions is higher than on $2 \mathrm{~L}-\mathrm{H}_{2} \mathrm{O}$ regions. A particular interesting detail in the c-AFM map is that there is a conductivity difference between the BL flake in the bottom right part of the map compared to the BL flake of the same thickness in the top left part of the map. This difference can be 


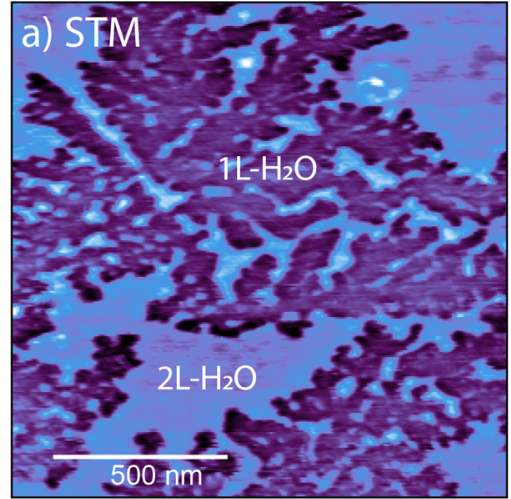

d)
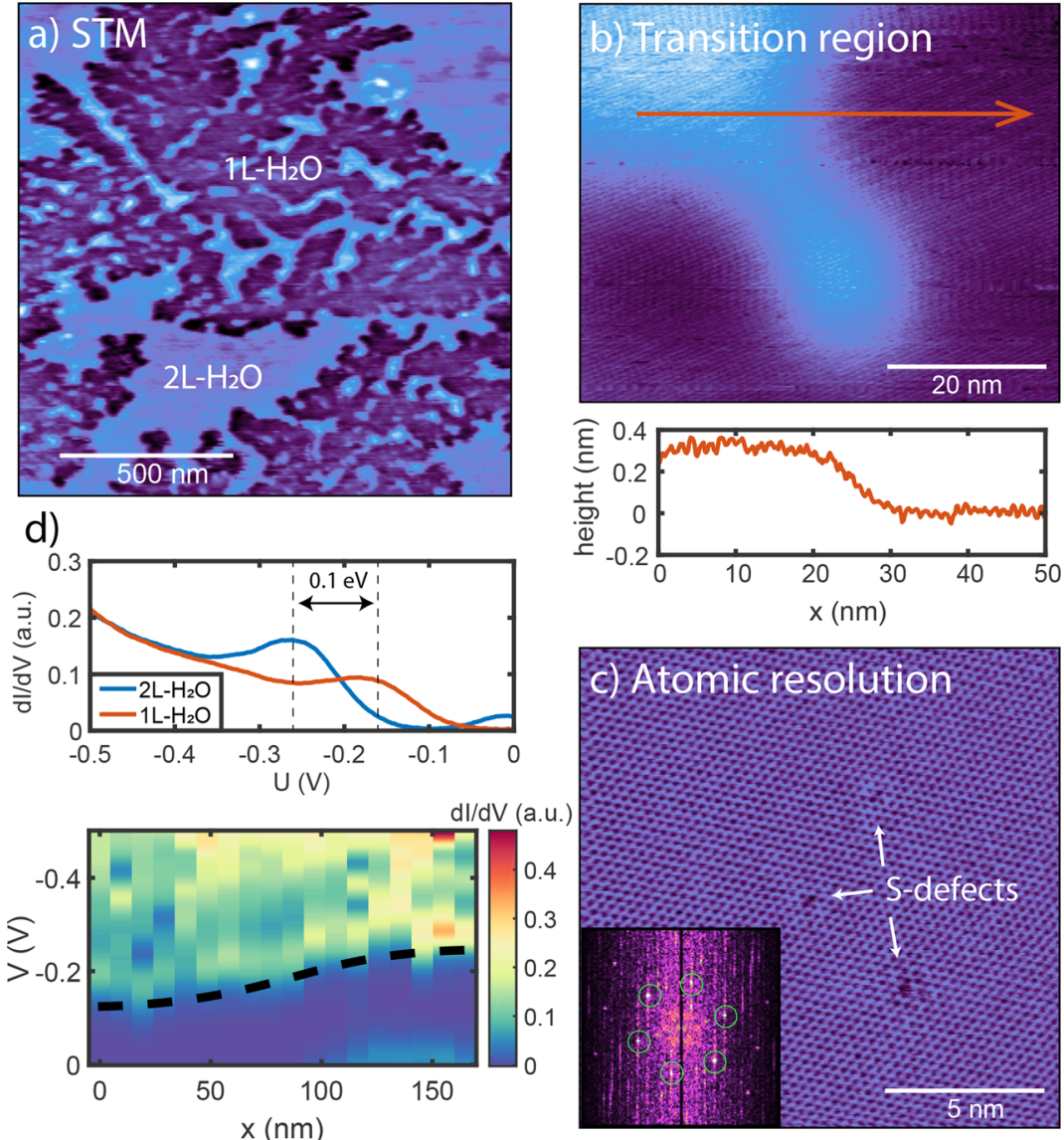

Figure 2. (a) STM topography image of an area with fractal shaped $1 \mathrm{~L}-\mathrm{H}_{2} \mathrm{O}$ regions surrounded by $2 \mathrm{~L}-\mathrm{H}_{2} \mathrm{O}$ regions $(V=-1.5 \mathrm{~V} ; \mathrm{I}=500 \mathrm{pA})$. (b) A smaller-scale topography map of the transition from a $2 \mathrm{~L}-\mathrm{H}_{2} \mathrm{O}$ region to a $1 \mathrm{~L}-\mathrm{H}_{2} \mathrm{O}$ region. The cross section is indicated by the red arrow $(V=$ $-1.0 \mathrm{~V} ; I=400 \mathrm{pA})$. (c) Atomically resolved image from within a $1 \mathrm{~L}-\mathrm{H}_{2} \mathrm{O}$ area $(V=-1.0 \mathrm{~V} ; I=400 \mathrm{pA})$. The Fourier transform of the lattice is shown in the inset, and the hexagonal symmetry of $\mathrm{WS}_{2}$ is indicated by the green circles. (d) $\mathrm{d} I(V) / \mathrm{d} V$ curves recorded on $\mathrm{WS}_{2} / 2 \mathrm{~L}-\mathrm{H}_{2} \mathrm{O}$ and $\mathrm{WS}_{2} /$ $1 \mathrm{~L}-\mathrm{H}_{2} \mathrm{O}$ regions. The shift of $0.1 \mathrm{~V}$ of a peak in the LDOS is indicated. At the bottom, a $\mathrm{d} I(V) / \mathrm{d} V$ cross section recorded across a transition region is shown. The black dashed line is a guide to the eye.

explained by the fact that the top layer of the BL part in the top left corner is partially isolated from the rest of the flake, as shown by the optical microscopy image in Figure $1 \mathrm{~b}$. This means that charge carriers cannot be transported laterally through the top layer and experience two contacts in series: one contact from the tip to the $\mathrm{WS}_{2}$ and a second contact from the top layer to the bottom layer. Because the $\mathrm{BL}$ region is relatively small, this second contact has a substantial contribution to the contact resistance, resulting in a lower conductivity. ${ }^{36}$

In order to determine why the contact properties differ between $2 \mathrm{~L}-\mathrm{H}_{2} \mathrm{O}$ and $1 \mathrm{~L}-\mathrm{H}_{2} \mathrm{O}$ regions, we have investigated the local work function variations over the sample $(\Phi)$ by Kelvin probe force microscopy (KPFM). $\Phi$ is calibrated on a thick $\mathrm{WS}_{2}$ flake to the known work function of bulk $\mathrm{WS}_{2}$ (as is described in more detail in the Supporting Information). ${ }^{41}$ As shown in Figure 1f, $\Phi$ is higher at the $\mathrm{WS}_{2} / 1 \mathrm{~L}-\mathrm{H}_{2} \mathrm{O}$ regions than at the $\mathrm{WS}_{2} / 2 \mathrm{~L}-\mathrm{H}_{2} \mathrm{O}$ regions. The work function difference between $\mathrm{WS}_{2} / 1 \mathrm{~L}-\mathrm{H}_{2} \mathrm{O}$ and $\mathrm{WS}_{2} / 2 \mathrm{~L}-\mathrm{H}_{2} \mathrm{O}$ is 0.11 $\mathrm{eV}$. The work function of the mica is $0.03 \mathrm{eV}$ higher than that of the $\mathrm{WS}_{2} / 2 \mathrm{~L}-\mathrm{H}_{2} \mathrm{O}$ region and $0.08 \mathrm{eV}$ lower than that of the $\mathrm{WS}_{2} / 1 \mathrm{~L}-\mathrm{H}_{2} \mathrm{O}$ region. A detailed description of how these average values are obtained from the measurement can be found in the Supporting Information, Figure S3. This shift in work function indicates p-type doping of $\mathrm{WS}_{2}$, similar to the findings for graphene. ${ }^{38,39}$ Previous work has shown that the thickness of the intercalated water film influences the capacitance of the total system. ${ }^{42}$ Here, the water layers are below the $\mathrm{WS}_{2}$ flake; thus, they do not (directly) influence the capacitance of the system. Another notable feature in Figure if is that $\Phi$ varies spatially within the fractals, increasing toward the center of the fractals. This nonuniform $\Phi$ can be attributed either to horizontal charge screening or to artifacts due to tipconvolution. It should be noted that the $\Phi$ decreases with increasing thickness of the $\mathrm{WS}_{2}$ flake, as shown in the KPFM map in Figure S2.

To study the topographic and electronic transition from the $1 \mathrm{~L}-\mathrm{H}_{2} \mathrm{O}$ to the $2 \mathrm{~L}-\mathrm{H}_{2} \mathrm{O}$ regions, we have performed scanning tunneling microscopy (STM) and scanning tunneling spectroscopy (STS) measurements. In Figure 2a, a topographic STM image of a FL WS $\mathrm{Wl}_{2}$ flake on mica is shown. Part of a large $1 \mathrm{~L}-\mathrm{H}_{2} \mathrm{O}$ fractal is captured within the recorded image. A smallscale STM image of $\mathrm{WS}_{2}$ of a border between a $1 \mathrm{~L}-\mathrm{H}_{2} \mathrm{O}$ region and a $2 \mathrm{~L}-\mathrm{H}_{2} \mathrm{O}$ region is shown in Figure $2 \mathrm{~b}$. The cross section of the transition reveals that the lattice periodicity of $\mathrm{WS}_{2}$ spans over both regions, unambiguously demonstrating that the fractal structure is located underneath the $\mathrm{WS}_{2}$ cover. Atomically resolved images of $\mathrm{WS}_{2}$ on the $1 \mathrm{~L}-\mathrm{H}_{2} \mathrm{O}$ regions, e.g., Figure 2c, reveal an almost perfect lattice with only a few sulfur defects (S-defects). The Fourier transform in the inset 
reveals the hexagonal periodicity of $\mathrm{WS}_{2}$. Figure $2 \mathrm{~d}$ shows $\mathrm{d} I(V) / \mathrm{d} V$ curves that are obtained from $I(V)$ traces recorded on $\mathrm{WS}_{2}$ on the $1 \mathrm{~L}-\mathrm{H}_{2} \mathrm{O}$ and $2 \mathrm{~L}-\mathrm{H}_{2} \mathrm{O}$ regions. By tracking the small peak in the density of states between $-0.1 \mathrm{~V}$ and $-0.3 \mathrm{~V}$, it is revealed that the valence band maximum (VBM) of the $\mathrm{WS}_{2}$ on the $1 \mathrm{~L}-\mathrm{H}_{2} \mathrm{O}$ is shifted to the right by about $0.1 \mathrm{eV}$ as compared to the VBM of $\mathrm{WS}_{2}$ on $2 \mathrm{~L}-\mathrm{H}_{2} \mathrm{O}$. This shift brings the Fermi level of the $\mathrm{WS}_{2} / 1 \mathrm{~L}-\mathrm{H}_{2} \mathrm{O}$ closer to the VBM, confirming p-type doping of the $\mathrm{WS}_{2}$, in line with the KPFM results. The full bias range of the $\mathrm{d} I(V) / \mathrm{d} V$ curves are shown in Figure S4. The measured $\mathrm{d} I(V) / \mathrm{d} V$ curves undergo a continuous transition from one region to the other, as can be seen by the $\mathrm{d} I(V) / \mathrm{d} V$ cross section to the bottom of the panel. The individual traces of the cross section are recorded over a transition region, as shown in detail in Figure S4.

The direct control of the doping level of the $\mathrm{WS}_{2}$ cover by manipulation of the underlying water structure is of particular interest in controlling the metal-semiconductor contact characteristics. First, we note that similar to the graphenemica system, ${ }^{32}$ wetting and dewetting of the interface are reversible and can be controlled by the $\mathrm{RH}$ of the environment as shown in Figure $3 a-d$. The scans are obtained at the same

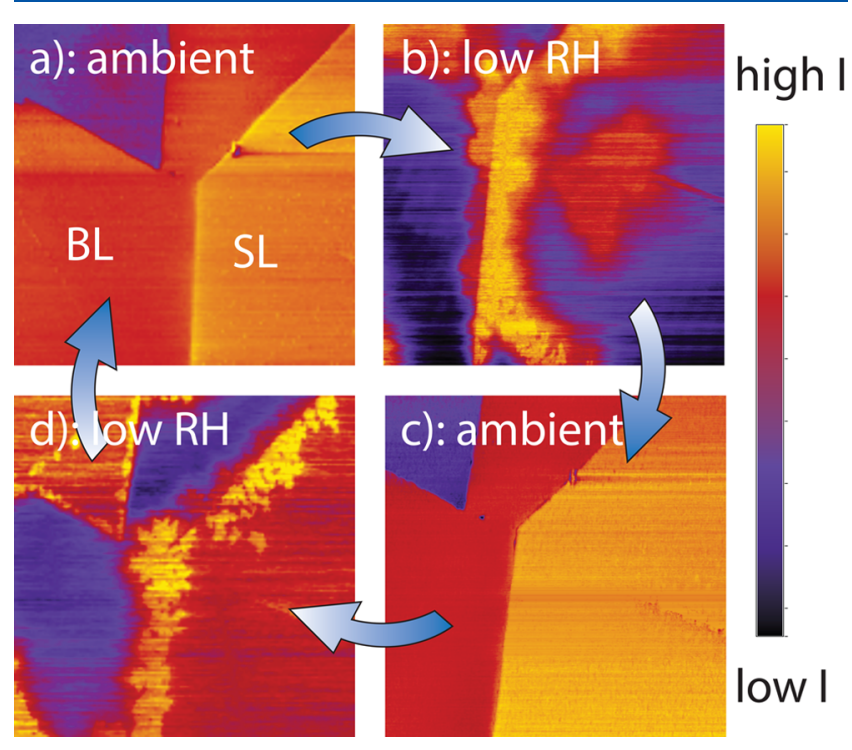

Figure 3. Four panels showing a current map of the same region. A bias voltage of $2.0 \mathrm{~V}$ was applied between the tip and sample. Through repeated decrease and increase of the $\mathrm{RH}$, water evaporates from and recondensates between the mica and $\mathrm{WS}_{2}$. (a) At ambient conditions, no fractals are visible in the current map. (b) After the $\mathrm{RH}$ is reduced, fractals start to grow where $2 \mathrm{~L}-\mathrm{H}_{2} \mathrm{O}$ reduces to $1 \mathrm{~L}-\mathrm{H}_{2} \mathrm{O}$, resulting in a local increase in current. (c) Exposing the system to ambient conditions restores the complete water film. (d) This process is completely reversible, and the fractals regrow upon another reduction of the $\mathrm{RH}$.

location as Figure 1c. By repeated changing of the environment from ambient conditions to low $\mathrm{RH}$, the water film will completely fill (Figure 3a), partly evaporate (Figure 3b), refill (Figure $3 \mathrm{c}$ ), and evaporate again (Figure $3 \mathrm{~d}$ ). It should be noted that the shape of the fractals at low $\mathrm{RH}$ can differ in every cycle. Nevertheless they provide regions where the charge carriers experience lower resistances, as is evident from the higher currents recorded at these sites. Controlling the charge doping and contact properties of future devices by intercalation of water (or molecules with different dipole strengths $)^{43}$ could provide a novel route to environmentally gate the $2 \mathrm{D}$ material. ${ }^{7,8}$ In the following, we will experimentally investigate the influence of the intercalated water on the charge carrier injection mechanism at the $\mathrm{Pt} / \mathrm{WS}_{2}$ contacts.

With c-AFM, the $I(V)$ characteristics of the $\mathrm{Pt} / \mathrm{WS}_{2}$ contact can be determined locally by placing the metallic tip on the $\mathrm{WS}_{2}$ and subsequently ramping the bias voltage. Because TE, $\mathrm{F}-\mathrm{N}$, and DT each have their own typical $I(V)$ characteristics, the recorded $I(V)$ curves can be linked to the dominant charge injection mechanism. In Figure $4 \mathrm{a}, I(V)$ curves are shown for contact locations on $\mathrm{WS}_{2}$ of different thicknesses: SL, BL, FL, and multilayer (ML) as schematically shown in Figure $4 \mathrm{~b}$. For each thickness, $\mathrm{I}(\mathrm{V})$ curves are recorded both on a $2 \mathrm{~L}-\mathrm{H}_{2} \mathrm{O}$ region as well as on a $1 \mathrm{~L}-\mathrm{H}_{2} \mathrm{O}$ region. The details of the acquirement of the $I(V)$ curves is presented in Figure S6. The conductivity of the recorded $I(V)$ curves depends on the layer thickness; the conductivity is lowest for $\mathrm{SL}_{-} \mathrm{WS}_{2}$, while it becomes higher for thicker $\mathrm{WS}_{2}$ flakes. This is in agreement with the c-AFM maps recorded in Figure 1e, where higher currents are obtained for thicker flakes. This thicknessdependent conductivity can be associated with either the thickness-dependent Schottky barrier height $(\mathrm{SBH})$ or a possible thickness-dependent change of the charge injection mechanism. $^{36,44}$

Moreover, it is noted that the conductivity on the $1 \mathrm{~L}-\mathrm{H}_{2} \mathrm{O}$ regions is higher at both positive and negative sample biases compared to the lower conductivity on the $2 \mathrm{~L}-\mathrm{H}_{2} \mathrm{O}$ regions. However, the difference in conductivity is larger at positive sample bias than at negative sample bias. Strikingly, the functional shape of the curves in Figure 4 a differs too. This indicates different injection mechanisms across the different contacts. $^{1-3}$ The three main injection mechanisms that we consider here are TE, F-N, and $\mathrm{DT}^{22} \mathrm{TE}$ is expected when electrons are thermally excited to overcome the Schottky barrier. For DT, electrons tunnel through an additional interfacial barrier which was found to be formed between van der Waals material and metal lead. ${ }^{1}$ DT becomes more prominent at lower temperatures and for thinner barriers. F-N typically occurs at higher bias voltages. For $\mathrm{F}-\mathrm{N}$, electrons tunnel through part of a triangular barrier. ${ }^{1}$ The three injection mechanisms are schematically described in Figure 4c.

TE is described by ${ }^{22,45}$

$$
I=I_{0} \exp \left(\frac{q V}{n k_{\mathrm{B}} T}\right)\left[1-\exp \left(-\frac{q V}{k_{\mathrm{B}} T}\right)\right]
$$

with $I_{0}$ the saturation current for TE equal to

$$
I_{0}=A A^{*} T^{2} \exp \left(-\frac{q \Phi_{\mathrm{B}}}{k_{\mathrm{B}} T}\right)
$$

where $V$ is the bias voltage, $T$ the temperature, $k_{\mathrm{B}}$ the Boltzmann constant, $q$ the elementary charge, $n$ the ideality factor, $A$ the electrical contact area, $A^{*}$ the Richardson constant, and $\Phi_{\mathrm{B}}$ the Schottky barrier height.

$\mathrm{DT}$ is described by ${ }^{22,46}$

$$
I=\frac{A q^{2} V \sqrt{2 m^{*} \Phi_{\mathrm{B}}}}{h^{2} d} \exp \left(-\frac{4 \pi d \sqrt{2 m^{*} \Phi_{\mathrm{B}}}}{h}\right)
$$

where $m^{*}$ is the effective mass of an electron $\left(0.31 \mathrm{~m}_{0},{ }^{47}\right.$ with $m_{0}$ the rest mass), $d$ the barrier width, and $h$ the Planck constant. 

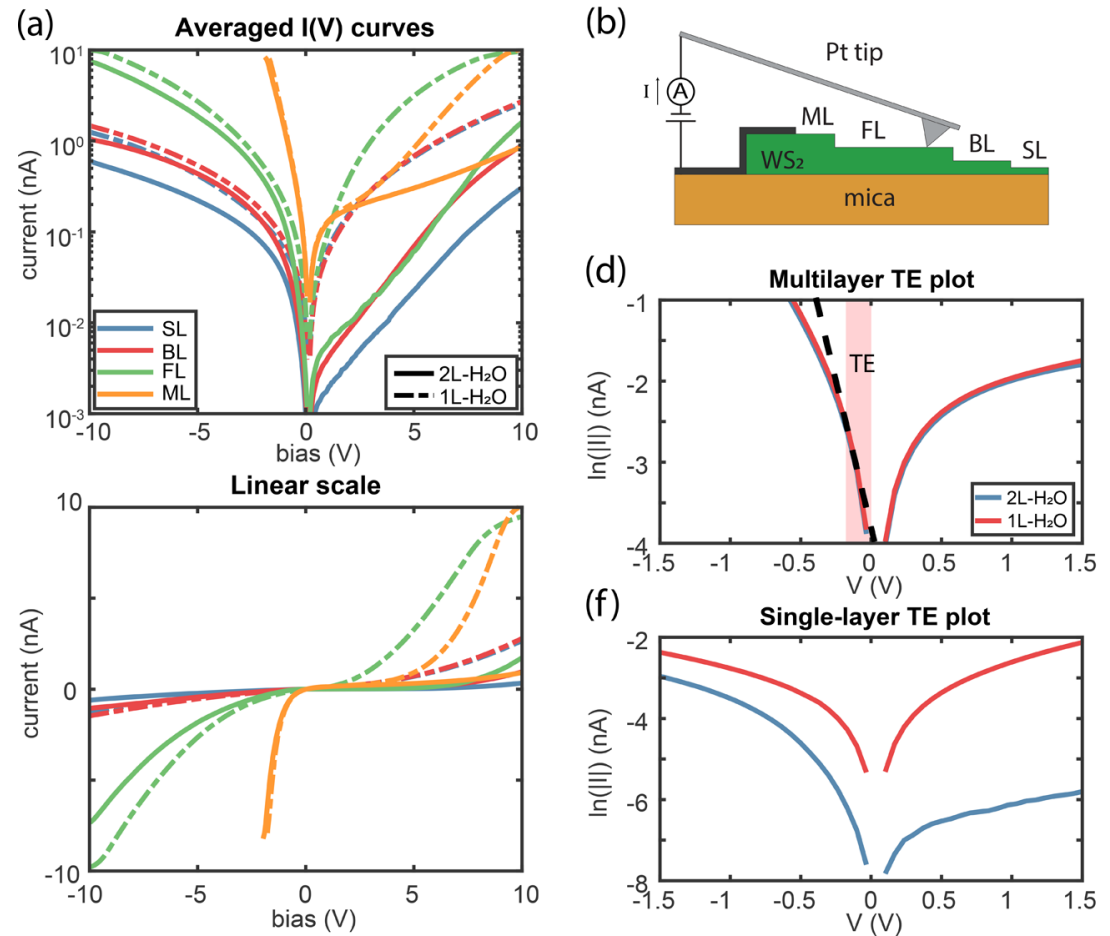

(c)
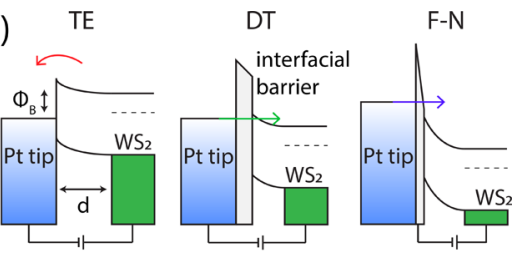

(e)

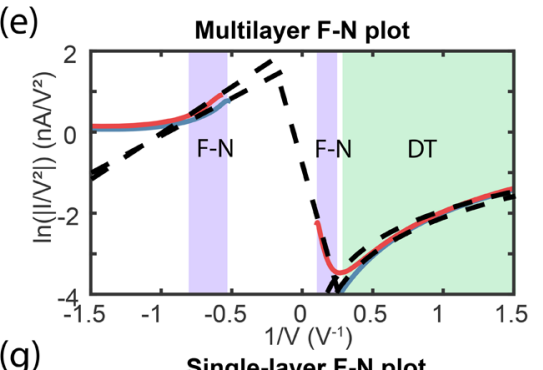

(g)

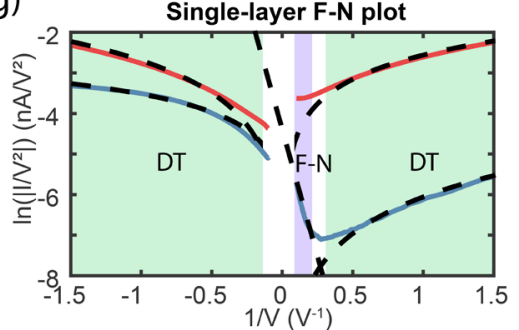

Figure 4. (a) Averaged $I(V)$ curves recorded by c-AFM on regions of different thickness from SL to BL, FL, and ML on $1 \mathrm{~L}-\mathrm{H}_{2} \mathrm{O}$ and $2 \mathrm{~L}-\mathrm{H}_{2} \mathrm{O}$. The lower panel shows the same data in a linear scale. (b) A side-view of the schematic setup used to obtain the data in panel a. (c) Schematic representation of the three injection mechanisms considered: TE, DT, and F-N. (d-g) The same data as in panel a for the ML and SL areas is plotted in logarithmic-linear ( $\mathrm{d}$ and f, respectively) and $\mathrm{F}-\mathrm{N}$ scales (e and g, respectively). The dashed lines are linear (TE, F-N) and logarithmic (DT) relations fitted to the data.

$\mathrm{F}-\mathrm{N}$ is described by ${ }^{22,46}$

$$
I=\frac{A q^{3} m_{0} V^{2}}{8 \pi h \Phi_{\mathrm{B}} d^{2} m^{*}} \exp \left[\frac{-8 \pi \sqrt{2 m^{*}} \Phi_{\mathrm{B}}{ }^{3 / 2} d}{3 h q V}\right]
$$

A careful investigation of the characteristic shape of the $I(V)$ traces can provide qualitative information on which of these injection mechanisms is dominant. TE results in a linear line when plotting $\ln (I)$ as a function of $V$ and in the bias range where $q V \gg 3 k_{\mathrm{B}} T .^{45}$ From the slope of the linear part that equals $q / n k_{B} T$, the ideality factor can be extracted (eq 1 ), giving a measure of the applicability of the model to describe the current transport by pure TE. F-N and DT can be distinguished when plotting $\ln \left(I / V^{2}\right)$ as a function of $1 / V(\mathrm{~F}-$ $\mathrm{N}$ plot). For $\mathrm{F}-\mathrm{N}$ tunneling, the curve will decrease linearly, whereas for DT, it should increase logarithmically. ${ }^{48,49}$ DT shows a linear behavior when plotted in a $\ln \left(I / V^{2}\right)$ versus $\ln ($ I $1 / \mathrm{VI})$ scale. $\mathrm{F}-\mathrm{N}$ is generally expected for higher applied bias voltages, while DT is expected for thin and small barriers. The two regimes are separated by an inflection point. ${ }^{48,49}$ For F-N and $\mathrm{DT}$, it is possible to determine the barrier parameters $\Phi_{\mathrm{B}}{ }^{3 / 2} d$ and $\sqrt{\Phi_{\mathrm{B}}} d$, respectively. The details of acquiring the parameters $\Phi_{\mathrm{B}}$ and $n$ for TE, $\Phi_{\mathrm{B}}^{3 / 2} d$ for F-N, and $\sqrt{\Phi_{\mathrm{B}}} d$ for DT are described in the Supporting Information.

To identify which injection mechanism dominates in different contacts, we have plotted the data acquired on SL$\mathrm{WS}_{2}$ and ML-WS ${ }_{2}$ in two different ways: $\ln (I)$ versus $V$ and $\ln \left(I / V^{2}\right)$ versus $1 / V$ in Figure $4 \mathrm{~d}-\mathrm{g}$. The data plotted in a $\ln \left(I / V^{2}\right)$ versus $\ln (|1 / V|)$ scale is shown in Figure S7. We first focus on the contact properties of ML-WS ${ }_{2}$. As shown in Figure 4d, the $I(V)$ curves are unipolar for both $\mathrm{ML}-\mathrm{WS}_{2}$ on
$1 \mathrm{~L}-\mathrm{H}_{2} \mathrm{O}$ as well as for ML-WS $\mathrm{S}_{2}$ on $2 \mathrm{~L}-\mathrm{H}_{2} \mathrm{O}$. In the forward bias regime (negative sample bias) and at low bias voltage, the data can be fitted well with the TE model, as shown in Figure 4d $(-0.3 \mathrm{~V}$ to $-0.05 \mathrm{~V})$. From TE we extract barrier heights of $1.1 \mathrm{eV}$ (both $1 \mathrm{~L}-\mathrm{H}_{2} \mathrm{O}$ and $2 \mathrm{~L}-\mathrm{H}_{2} \mathrm{O}$ ) and ideality factors equal to 5.5 (both $1 \mathrm{~L}-\mathrm{H}_{2} \mathrm{O}$ and $2 \mathrm{~L}-\mathrm{H}_{2} \mathrm{O}$ ). The injection mechanisms at higher forward biases and at reverse biases are dominated by $\mathrm{F}-\mathrm{N}$ and DT, as can be seen in Figure 4e. At high forward bias $(<-1.2 \mathrm{~V})$, both the $1 \mathrm{~L}-\mathrm{H}_{2} \mathrm{O}$ and $2 \mathrm{~L}-\mathrm{H}_{2} \mathrm{O}$ regions can be described by $\mathrm{F}-\mathrm{N}$ with barrier parameters of $0.60 \mathrm{eV}^{3 / 2} \mathrm{~nm}$ $\left(1 \mathrm{~L}-\mathrm{H}_{2} \mathrm{O}\right)$ and $0.49 \mathrm{eV}^{3 / 2} \mathrm{~nm}\left(2 \mathrm{~L}-\mathrm{H}_{2} \mathrm{O}\right)$. In the reverse bias regime, the dominating injection mechanism depends on the hydration state of the substrate. In a $1 \mathrm{~L}-\mathrm{H}_{2} \mathrm{O}$ region, the $\mathrm{F}-\mathrm{N}$ plot reveals a clear inflection point with a linear decreasing behavior at high voltages $(\mathrm{F}-\mathrm{N})$ and a logarithmic increasing behavior at lower voltages (DT).$^{48}$ The barrier parameter of the $\mathrm{DT}$ regime is $0.34 \mathrm{eV}^{1 / 2} \mathrm{~nm}$, while in the $\mathrm{F}-\mathrm{N}$ regime the barrier parameter is $3.27 \mathrm{eV}^{3 / 2} \mathrm{~nm}$. In the $2 \mathrm{~L}-\mathrm{H}_{2} \mathrm{O}$ region, on the other hand, the injection mechanism is DT over the full reverse bias range with a barrier parameter of $0.37 \mathrm{eV}^{1 / 2} \mathrm{~nm}$.

On SL-WS ${ }_{2}$, we see a much different behavior. While the Pt/ $\mathrm{SL}-\mathrm{WS}_{2} / 2 \mathrm{~L}-\mathrm{H}_{2} \mathrm{O}$ contact is unipolar, the Pt $/ \mathrm{SL}-\mathrm{WS}_{2} / 1 \mathrm{~L}-\mathrm{H}_{2} \mathrm{O}$ contact shows an ambipolar behavior. This change in contact properties can be explained by the different injection mechanisms that are dominant at SL-WS ${ }_{2}$, as can be seen in Figures 4f,g. In Figure 4f, no linear trend is found at low bias voltages, indicating that the curves can not be described properly by TE. Meanwhile, in Figure $4 \mathrm{~g}$ it is shown that at lower voltages the $I(V)$ traces are well described by DT with barrier parameters of $0.48 \mathrm{eV}^{1 / 2} \mathrm{~nm}\left(1 \mathrm{~L}-\mathrm{H}_{2} \mathrm{O}\right.$, forward bias), $0.45 \mathrm{eV}^{1 / 2} \mathrm{~nm}\left(1 \mathrm{~L}-\mathrm{H}_{2} \mathrm{O}\right.$, reverse bias), $0.62 \mathrm{eV}^{1 / 2} \mathrm{~nm}(2 \mathrm{~L}-$ $\mathrm{H}_{2} \mathrm{O}$, forward bias), and $1.06 \mathrm{eV}^{1 / 2} \mathrm{~nm}\left(2 \mathrm{~L}-\mathrm{H}_{2} \mathrm{O}\right.$, reverse bias). 
At higher bias voltages in the reverse bias regime $(V>5 \mathrm{~V})$ at $2 \mathrm{~L}-\mathrm{H}_{2} \mathrm{O}$, a clear $\mathrm{F}-\mathrm{N}$ contribution can be distinguished with a barrier parameter of $3.28 \mathrm{eV}^{3 / 2} \mathrm{~nm}$. Comparing $1 \mathrm{~L}-\mathrm{H}_{2} \mathrm{O}$ to $2 \mathrm{~L}$ $\mathrm{H}_{2} \mathrm{O}$, the current at the reverse bias regime is substantially increased, changing the behavior of the device from unipolar to ambipolar. The BL and FL results show that the dominant charge injection mechanisms are the same as for the case of SL $\mathrm{WS}_{2}$ as is shown in Figure S7. Still, the measured barrier parameters change for thicker parts of the flake. The barrier parameters for DT decrease overall for increasing flake thickness, as can be seen in Table 1, which explains the increase in conductivity.

Table 1. Barrier Parameters for Thin $\mathrm{WS}_{2}$

\begin{tabular}{lccc} 
& SL & BL & FL \\
F-N, 2L- $\mathrm{H}_{2} \mathrm{O}$ reverse $\left(\mathrm{eV}^{3 / 2} \mathrm{~nm}\right)$ & 3.28 & 3.04 & 5.23 \\
$\mathrm{DT}, 2 \mathrm{~L}-\mathrm{H}_{2} \mathrm{O}$ forward $\left(\mathrm{eV}^{1 / 2} \mathrm{~nm}\right)$ & 0.62 & 0.54 & 0.39 \\
$\mathrm{DT}, 2 \mathrm{~L}-\mathrm{H}_{2} \mathrm{O}$ reverse $\left(\mathrm{eV}^{1 / 2} \mathrm{~nm}\right)$ & 1.06 & 0.93 & 0.87 \\
DT, 1L- $\mathrm{H}_{2} \mathrm{O}$ forward $\left(\mathrm{eV}^{1 / 2} \mathrm{~nm}\right)$ & 0.48 & 0.45 & 0.28 \\
DT, 1L- $\mathrm{H}_{2} \mathrm{O}$ reverse $\left(\mathrm{eV}^{1 / 2} \mathrm{~nm}\right)$ & 0.45 & 0.47 & 0.35 \\
\hline
\end{tabular}

The results obtained here are also of interest for larger macroscopic contacts to $\mathrm{WS}_{2}$ on mica (or any $2 \mathrm{D}$ semiconductor on a hydrophilic substrate). At low $\mathrm{RH}$, the charge carrier injection through the contact will occur at the $1 \mathrm{~L}-\mathrm{H}_{2} \mathrm{O}$ regions, as the resistance is the lowest in these regions. Exposing the system to ambient $\mathrm{RH}$ leads to filling of the fractals and the completion of the second $\mathrm{H}_{2} \mathrm{O}$ layer. The contact properties will now be determined by the injection mechanism on $2 \mathrm{~L}-\mathrm{H}_{2} \mathrm{O}$. Switching between injection mechanisms and switching between unipolar and ambipolar transport by controlling the $\mathrm{RH}$ is therefore also possible for macroscopic contacts.

In conclusion, we have demonstrated that by controlling the hydration state of the underlying supporting substrate it is possible to modify the charge carrier injection mechanism in $\mathrm{WS}_{2}-$ metal nanocontacts. This environmentally controlled process is the consequence of doping due to charge transfer from the underlying water layer. In addition, the charge injection mechanism strongly depends on the thickness of the $\mathrm{WS}_{2}$ flake. TE was observed only on $\mathrm{ML} \mathrm{WS}$, whereas for SL, $\mathrm{BL}$, and $\mathrm{FL} \mathrm{WS}$, a switch from unipolar to ambipolar behavior was observed when decreasing the thickness of the intercalated water from $2 \mathrm{~L}$ to $1 \mathrm{~L}$. The reversibility of the dewetting process along with its noninvasive character provides a viable alternative for fabricating devices with in situ control of the metal-semiconductor junction characteristics.

\section{ASSOCIATED CONTENT}

\section{S Supporting Information}

The Supporting Information is available free of charge on the ACS Publications website at DOI: 10.1021/acs.jpclett.9b00862.

Methods section and details on the acquirement of the c-AFM $I(V)$ curves, thickness-dependent work function, KPFM analysis, acquirement of the STM data, and the carrier injection mechanisms. (PDF)

\section{AUTHOR INFORMATION}

\section{Corresponding Authors}

*E-mail: r.vanbremen@utwente.nl.
*E-mail: bampoulis@ph2.uni-koeln.de.

ORCID

Rik van Bremen: 0000-0002-2088-1568

Pantelis Bampoulis: 0000-0002-2347-5223

Notes

The authors declare no competing financial interest.

\section{ACKNOWLEDGMENTS}

R.v.B., H.J.W.Z., and P.B. thank the Dutch organization for scientific research NWO for financial support (FV157 14TWDO07). P.B. thanks the Alexander von Humboldt foundation for financial support.

\section{REFERENCES}

(1) Ahmed, F.; Choi, M. S.; Liu, X.; Yoo, W. J. Carrier Transport at the Metal-MoS2 Interface. Nanoscale 2015, 7, 9222-9228.

(2) Liu, H.; Si, M.; Deng, Y.; Neal, A. T.; Du, Y.; Najmaei, S.; Ajayan, P. M.; Lou, J.; Ye, P. D. Switching Mechanism in Single-Layer Molybdenum Disulfide Transistors: An Insight into Current Flow across Schottky Barriers. ACS Nano 2014, 8, 1031-1038.

(3) Allain, A.; Kang, J.; Banerjee, K.; Kis, A. Electrical Contacts to Two-Dimensional Semiconductors. Nat. Mater. 2015, 14, 11951205.

(4) Schulman, D. S.; Arnold, A. J.; Das, S. Contact Engineering for 2D Materials and Devices. Chem. Soc. Rev. 2018, 47, 3037-3058.

(5) Schultz, B. J.; Jaye, C.; Lysaght, P. S.; Fischer, D. A.; Prendergast, D.; Banerjee, S. On Chemical Bonding and Electronic Structure of Graphene-Metal Contacts. Chemical Science 2013, 4, 494-502.

(6) Wang, Y.; Kim, J. C.; Wu, R. J.; Martinez, J.; Song, X.; Yang, J.; Zhao, F.; Mkhoyan, A.; Jeong, H. Y.; Chhowalla, M. Van Der Waals Contacts between Three-Dimensional Metals and Two-Dimensional Semiconductors. Nature 2019, 568, 70.

(7) Lee, S. Y.; Kim, U. J.; Chung, J.; Nam, H.; Jeong, H. Y.; Han, G. H.; Kim, H.; Oh, H. M.; Lee, H.; Kim, H.; et al. Large Work Function Modulation of Monolayer MoS2 by Ambient Gases. ACS Nano 2016, $10,6100-6107$.

(8) Tongay, S.; Zhou, J.; Ataca, C.; Liu, J.; Kang, J. S.; Matthews, T. S.; You, L.; Li, J.; Grossman, J. C.; Wu, J. Broad-Range Modulation of Light Emission in Two-Dimensional Semiconductors by Molecular Physisorption Gating. Nano Lett. 2013, 13, 2831-2836.

(9) Politano, A.; Chiarello, G.; Samnakay, R.; Liu, G.; Gürbulak, B.; Duman, S.; Balandin, A. A.; Boukhvalov, D. W. The Influence of Chemical Reactivity of Surface Defects on Ambient-Stable InSe-Based Nanodevices. Nanoscale 2016, 8, 8474-8479.

(10) Viti, L.; Politano, A.; Vitiello, M. S. Black Phosphorus Nanodevices at Terahertz Frequencies: Photodetectors and Future Challenges. APL Mater. 2017, 5, 035602.

(11) Politano, A.; Chiarello, G. Unravelling Suitable GrapheneMetal Contacts for Graphene-Based Plasmonic Devices. Nanoscale 2013, 5, 8215-8220.

(12) Kim, C.; Moon, I.; Lee, D.; Choi, M. S.; Ahmed, F.; Nam, S.; Cho, Y.; Shin, H.-J.; Park, S.; Yoo, W. J. Fermi Level Pinning at Electrical Metal Contacts of Monolayer Molybdenum Dichalcogenides. ACS Nano 2017, 11, 1588-1596.

(13) Sotthewes, K.; van Bremen, R.; Dollekamp, E.; Boulogne, T.; Nowakowski, K.; Kas, D.; Zandvliet, H. J. W.; Bampoulis, P. Universal Fermi-Level Pinning in Transition-Metal Dichalcogenides. J. Phys. Chem. C 2019, 123, 5411-5420.

(14) Das, S.; Chen, H.-Y.; Penumatcha, A. V.; Appenzeller, J. High Performance Multilayer MoS2 Transistors with Scandium Contacts. Nano Lett. 2013, 13, 100-105.

(15) Liu, Y.; Guo, J.; Zhu, E.; Liao, L.; Lee, S.-J.; Ding, M.; Shakir, I.; Gambin, V.; Huang, Y.; Duan, X. Approaching the Schottky-Mott Limit in van Der Waals Metal-Semiconductor Junctions. Nature 2018, 557, 696.

(16) Liu, Y.; Wu, H.; Cheng, H.-C.; Yang, S.; Zhu, E.; He, Q.; Ding, M.; Li, D.; Guo, J.; Weiss, N. O.; et al. Toward Barrier Free Contact 
to Molybdenum Disulfide Using Graphene Electrodes. Nano Lett. 2015, 15, 3030-3034.

(17) Cui, X.; Shih, E.-M.; Jauregui, L. A.; Chae, S. H.; Kim, Y. D.; Li, B.; Seo, D.; Pistunova, K.; Yin, J.; Park, J.-H.; et al. Low-Temperature Ohmic Contact to Monolayer MoS2 by van Der Waals Bonded Co/hBN Electrodes. Nano Lett. 2017, 17, 4781-4786.

(18) Nowakowski, K.; van Bremen, R.; Zandvliet, H. J. W.; Bampoulis, P. Control of the Metal/WS2 Contact Properties Using 2-Dimensional Buffer Layers. Nanoscale 2019, 11, 5548-5556.

(19) English, C. D.; Shine, G.; Dorgan, V. E.; Saraswat, K. C.; Pop, E. Improved Contacts to MoS2 Transistors by Ultra-High Vacuum Metal Deposition. Nano Lett. 2016, 16, 3824-3830.

(20) Baugher, B. W. H.; Churchill, H. O. H.; Yang, Y.; JarilloHerrero, P. Intrinsic Electronic Transport Properties of High-Quality Monolayer and Bilayer MoS2. Nano Lett. 2013, 13, 4212-4216.

(21) Khalil, H. M. W.; Khan, M. F.; Eom, J.; Noh, H. Highly Stable and Tunable Chemical Doping of Multilayer WS2 Field Effect Transistor: Reduction in Contact Resistance. ACS Appl. Mater. Interfaces 2015, 7, 23589-23596.

(22) Durán Retamal, J. R.; Periyanagounder, D.; Ke, J.-J.; Tsai, M.L.; He, J.-H. Charge Carrier Injection and Transport Engineering in Two-Dimensional Transition Metal Dichalcogenides. Chemical Science 2018, 9, 7727-7745.

(23) Kuc, A.; Zibouche, N.; Heine, T. Influence of Quantum Confinement on the Electronic Structure of the Transition Metal Sulfide $\mathrm{TS}_{2}$. Phys. Rev. B: Condens. Matter Mater. Phys. 2011, 83, 245213.

(24) Ovchinnikov, D.; Allain, A.; Huang, Y.-S.; Dumcenco, D.; Kis, A. Electrical Transport Properties of Single-Layer $\mathrm{WS}_{2}$. ACS Nano 2014, 8, 8174-8181.

(25) Withers, F.; Bointon, T. H.; Hudson, D. C.; Craciun, M. F.; Russo, S. Electron Transport of $\mathrm{WS}_{2}$ Transistors in a Hexagonal Boron Nitride Dielectric Environment. Sci. Rep. 2015, 4, 4967.

(26) Samaddar, S.; Yudhistira, I.; Adam, S.; Courtois, H.; Winkelmann, C. B. Charge Puddles in Graphene near the Dirac Point. Phys. Rev. Lett. 2016, 116, 126804.

(27) Lui, C. H.; Liu, L.; Mak, K. F.; Flynn, G. W.; Heinz, T. F. Ultraflat Graphene. Nature 2009, 462, 339-341.

(28) Low, C. G.; Zhang, Q.; Hao, Y.; Ruoff, R. S. Graphene Field Effect Transistors with Mica as Gate Dielectric Layers. Small 2014, 10, 4213-4218.

(29) Verdaguer, A.; Sacha, G. M.; Bluhm, H.; Salmeron, M. Molecular Structure of Water at Interfaces: Wetting at the Nanometer Scale. Chem. Rev. 2006, 106, 1478-1510.

(30) Bampoulis, P.; Sotthewes, K.; Dollekamp, E.; Poelsema, B. Water Confined in Two-Dimensions: Fundamentals and Applications. Surf. Sci. Rep. 2018, 73, 233-264.

(31) Xu, K.; Cao, P.; Heath, J. R. Graphene Visualizes the First Water Adlayers on Mica at Ambient Conditions. Science 2010, 329, $1188-1191$

(32) Severin, N.; Lange, P.; Sokolov, I. M.; Rabe, J. P. Reversible Dewetting of a Molecularly Thin Fluid Water Film in a Soft Graphene-Mica Slit Pore. Nano Lett. 2012, 12, 774-779.

(33) He, K. T.; Wood, J. D.; Doidge, G. P.; Pop, E.; Lyding, J. W. Scanning Tunneling Microscopy Study and Nanomanipulation of Graphene-Coated Water on Mica. Nano Lett. 2012, 12, 2665-2672.

(34) Song, J.; Li, Q.; Wang, X.; Li, J.; Zhang, S.; Kjems, J.; Besenbacher, F.; Dong, M. Evidence of Stranski-Krastanov Growth at the Initial Stage of Atmospheric Water Condensation. Nat. Commun. 2014, 5, 4837.

(35) Dollekamp, E.; Bampoulis, P.; Faasen, D. P.; Zandvliet, H. J. W.; Kooij, E. S. Charge Induced Dynamics of Water in a GrapheneMica Slit Pore. Langmuir 2017, 33, 11977-11985.

(36) Pam, M. E.; Li, Z.; Ang, Y. S.; Shi, Y.; Geng, D.; Huang, S.; Zhao, X.; Pennycook, S. J.; Yao, H.; Gong, X.; et al. Thermal-Assisted Vertical Electron Injections in Few-Layer Pyramidal-Structured MoS2 Crystals. J. Phys. Chem. Lett. 2019, 10, 1292-1299.
(37) Goncher, S. J.; Zhao, L.; Pasupathy, A. N.; Flynn, G. W. Substrate Level Control of the Local Doping in Graphene. Nano Lett. 2013, 13, 1386-1392.

(38) Bampoulis, P.; Siekman, M. H.; Kooij, E. S.; Lohse, D.; Zandvliet, H. J. W.; Poelsema, B. Latent Heat Induced Rotation Limited Aggregation in 2D Ice Nanocrystals. J. Chem. Phys. 2015, 143, 034702 .

(39) Shim, J.; Lui, C. H.; Ko, T. Y.; Yu, Y.-J.; Kim, P.; Heinz, T. F.; Ryu, S. Water-Gated Charge Doping of Graphene Induced by Mica Substrates. Nano Lett. 2012, 12, 648-654.

(40) Bampoulis, P.; van Bremen, R.; Yao, Q.; Poelsema, B.; Zandvliet, H. J. W.; Sotthewes, K. Defect Dominated Charge Transport and Fermi Level Pinning in MoS2/Metal Contacts. ACS Appl. Mater. Interfaces 2017, 9, 19278-19286.

(41) Britnell, L.; Ribeiro, R. M.; Eckmann, A.; Jalil, R.; Belle, B. D.; Mishchenko, A.; Kim, Y.-J.; Gorbachev, R. V.; Georgiou, T.; Morozov, S. V.; et al. Strong Light-Matter Interactions in Heterostructures of Atomically Thin Films. Science 2013, 340, 1311-1314.

(42) Olson, E. J.; Ma, R.; Sun, T.; Ebrish, M. A.; Haratipour, N.; Min, K.; Aluru, N. R.; Koester, S. J. Capacitive Sensing of Intercalated $\mathrm{H} 2 \mathrm{O}$ Molecules Using Graphene. ACS Appl. Mater. Interfaces 2015, 7, 25804-25812.

(43) Kim, H. H.; Yang, J. W.; Jo, S. B.; Kang, B.; Lee, S. K.; Bong, H.; Lee, G.; Kim, K. S.; Cho, K. Substrate-Induced Solvent Intercalation for Stable Graphene Doping. ACS Nano 2013, 7, $1155-1162$

(44) Li, H.-M.; Lee, D.; Qu, D.; Liu, X.; Ryu, J.; Seabaugh, A.; Yoo, W. J. Ultimate Thin Vertical $\mathrm{p}-\mathrm{n}$ Junction Composed of TwoDimensional Layered Molybdenum Disulfide. Nat. Commun. 2015, 6, 6564.

(45) Rhoderick, E. H. Metal-Semiconductor Contacts. IEE Proc., Part I: Solid-State Electron Devices 1982, 129, 1-14.

(46) Das, S.; Prakash, A.; Salazar, R.; Appenzeller, J. Toward LowPower Electronics: Tunneling Phenomena in Transition Metal Dichalcogenides. ACS Nano 2014, 8, 1681-1689.

(47) Jin, Z.; Li, X.; Mullen, J. T.; Kim, K. W. Intrinsic Transport Properties of Electrons and Holes in Monolayer Transition-Metal Dichalcogenides. Phys. Rev. B: Condens. Matter Mater. Phys. 2014, 90, 045422 .

(48) Beebe, J. M.; Kim, B.; Gadzuk, J. W.; Daniel Frisbie, C.; Kushmerick, J. G. Transition from Direct Tunneling to Field Emission in Metal-Molecule-Metal Junctions. Phys. Rev. Lett. 2006, 97, 026801.

(49) Ikuno, T.; Okamoto, H.; Sugiyama, Y.; Nakano, H.; Yamada, F.; Kamiya, I. Electron Transport Properties of Si Nanosheets: Transition from Direct Tunneling to Fowler-Nordheim Tunneling. Appl. Phys. Lett. 2011, 99, 023107. 